\title{
Choi-Williams time-frequency analysis of forearm flexors Mechanomyogram acquired from a laser diode sensor based probe
}

\author{
S. Mekaoui ${ }^{1,2}$, K. Ghoumid ${ }^{2,3} \&$ T. Gharbi ${ }^{2}$ \\ ${ }^{1}$ Département des Télécommunications, Algérie \\ ${ }^{2}$ Institut des Microtechniques, Laboratoire d'Optique, \\ Pierre Marie Duffieux, UFR, Besançon, France \\ ${ }^{3} E N S A O$, Laboratoire d'Electronique et Télécommunication, \\ Complexe Universitaire, Oujda, Maroc
}

\begin{abstract}
The aim of this paper is to show how time-frequency plane can be used to directly read the frequency contents of forearm flexors muscles vibrations actually called MMG signals (Mechanomyogram). The MMG signals under scope are acquired from a laser diode sensor based probe connected to precise interface acquisition cards and the acquired signals are put into the memory of a PC for further analysis. The time-frequency technique used is the Choi-Williams energy distribution which belongs to the well reputed Cohen's class and satisfies many very useful properties especially in its $3 \mathrm{D}$ version. With this technique a good compromise between time and frequency resolution can be found. The 3D spectrograms displayed from the data acquired from few normal subjects and patients with specific muscle disorders are compared. This analysis method revealed an efficient localization of the MMG most significant frequencies.

Keywords: $M M G$ signals (Mechanomyogram), optical laser diode sensor, time-frequency analysis, Choi-Williams distribution, Morlet Wavelet Scalograms.
\end{abstract}

\section{Introduction}

Nowadays, a considerable progress has been performed in developing various methods for the acquisition and analysis of MMG signal (Mechanomyogram). In 
this experimentation, measurements of Mechanomyogram have been performed thanks to a specific laser diode sensor based probe. This system has been designed and realized by our team at Pierre Marie Duffieux laboratories [1]. Intensive MMG measurements has been made on forearm flexors muscles of normal subjects and patients fulfilling the steps and the requirements of a specific exercise protocol defined by D.T. Barry and recalled by C. Orizio [2, 3]. Whatever be the category of the tested subject, he is sat so that he can produce an isometric contraction to foresee with this experimentation the dynamic properties of the tested muscles $[2,3]$. The patients were tested under their consent and the agreement of the relevant hospital authorities.

.....The second objective of this work is to analyze and treat the Mechanomyogram to determine the frequency contents of such biomedical signals. Unfortunately, this class of signals is random and non stationary and it appears that specific methods of analysis are required. Among these various methods, Time-Frequency Analysis seems to be the most suitable and powerful tool to the treatment of such a kind of signals especially when the acquisition is made with an optical laser beam probe [1,3-6]. In this work, we have chosen the well known Choi-Williams energy distribution [7], which belongs to the very reputed Cohen's Class of energy distributions [8]. Although this technique presents noticeable remaining negative cross terms in the time frequency plane which can blur the readability of the 3D time frequency representation, it offers a good compromise between time and frequency resolutions and hence allows a good localization of the most significant frequency components of the MMG [7-9].

Many authors have shown how to improve time frequency distributions to perfectly localize the frequency contents of the analyzed signals and specifically those who worked in the time scale plane with reassigned wavelet scalograms $[3,4,9]$. We, here, are comparing the efficiency of the time frequency ChoiWilliams spectrograms with the known reassigned wavelet scalograms obtained on same tested muscles from same normal subjects and patients.

\section{Materials and methods}

\subsection{Description of the principle of measurements}

Figure 1 below, explicitly illustrates the principle of the optical measurement of the MMG signal.

This home probe was designed and realized to be suitable to normal subject and goodly adapted to patients affected by severe muscles diseases [2]. It had been especially immunized from any source of hazard shock [3], to be safely usable by the physicians in clinical environment. The laser diode used is a sharp of a LTO $26 \mathrm{MDO}(785 \mathrm{~nm}, 3 \mathrm{~mW})$ type and the measurements is based on the backscattered light beam on the skin. The reflected laser beam goes into the laser cavity and the backscattering beam modulated by the low frequency signal of interest (e.g. MMG Signal) is then captured. The target (studied muscle) is 


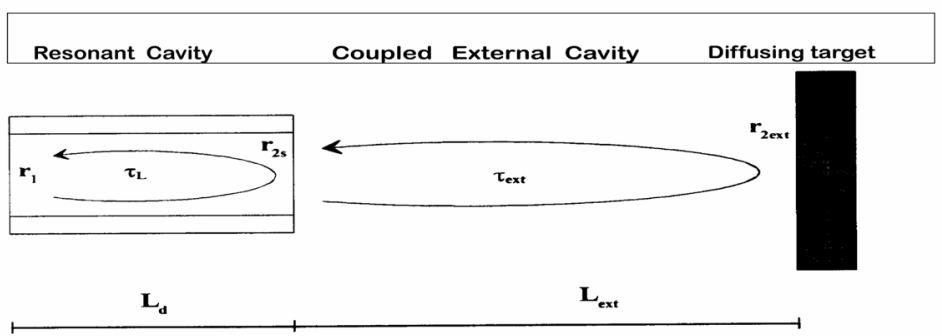

Figure 1: Measurement principle of the MMG Signals by the re-injection of the back-scattered laser light into the laser diode cavity, where: $\mathbf{L}_{\mathbf{d}}$ : is the length of the internal diode cavity, $\mathbf{L}_{\mathbf{e x t}}$ : is the length of the external cavity, $\boldsymbol{\tau}_{\mathbf{L}}$ and $\boldsymbol{\tau}_{\text {ext }}$ are the wave round trip times respectively in the diode cavity and in the coupled external cavity, $\mathbf{r}_{1}$ and $\mathbf{r}_{2 \mathrm{~s}}$ are the reflection coefficients respectively for the backward and forward faces of the laser diode, $\mathbf{r}_{\mathbf{2} \text { ext }}$ is the reflection coefficient of the target.

placed at an adjustable distance $\mathrm{L}_{\text {ext }}$ and the subject sat in such a way that he can produce an isometric contraction when grasping a specific strain gauge containing a strength sensor which measures simultaneously the force signal in newtons of his voluntary contraction. This simultaneous joint system is depicted in reference 2. It is well known that laser diodes are very sensitive to optical feedback. The re-injected power even extremely small is enough to perturb and vary the emission. In this case, according to Peterman [5] and to what had been described in references $[2,6]$, it can be mathematically proved that the final expression of the optical power $\Delta \mathrm{P}$ due to the current re-injection into the laser diode can simply be expressed by:

$$
\Delta \mathrm{P}=\Delta \mathrm{P}_{\max } \cos \left(\omega_{\mathrm{c}} \tau_{\mathrm{ext}}\right) ;
$$

where, $\Delta \mathrm{P}_{\max }$ is the maximum variation of the power due to the re-injection current in the internal cavity of the laser diode,

$\omega_{\mathrm{c}}$ : is the angular velocity in the laser cavity.

One should refer to reference 2 to find the mathematical proof of equation (1).

Figure 2 hereafter, gives the block diagram which details how the power variation $\Delta \mathrm{P}$ is captured with the Low Frequency MMG signal (BF) superimposed on it from the backscattered High Frequency beam (HF) reentering the laser diode cavity.

The beam is focused on the skin with the help of a controlled $200 \mathrm{~mm}$ focal length lens from a designed control system added to the probe. The injection current in the diode is modulated by a $2.5 \mathrm{~m}$ A symmetric triangular $500 \mathrm{~Hz}$ signal. The optical power $\mathrm{P}$ is measured by the mean of a photodiode incorporated in the laser package and then $\triangle \mathrm{P}$ containing the $\mathrm{MMG}$ signal of interest is in a first step filtered out from $\mathrm{P}$ and saved with the superimposed 


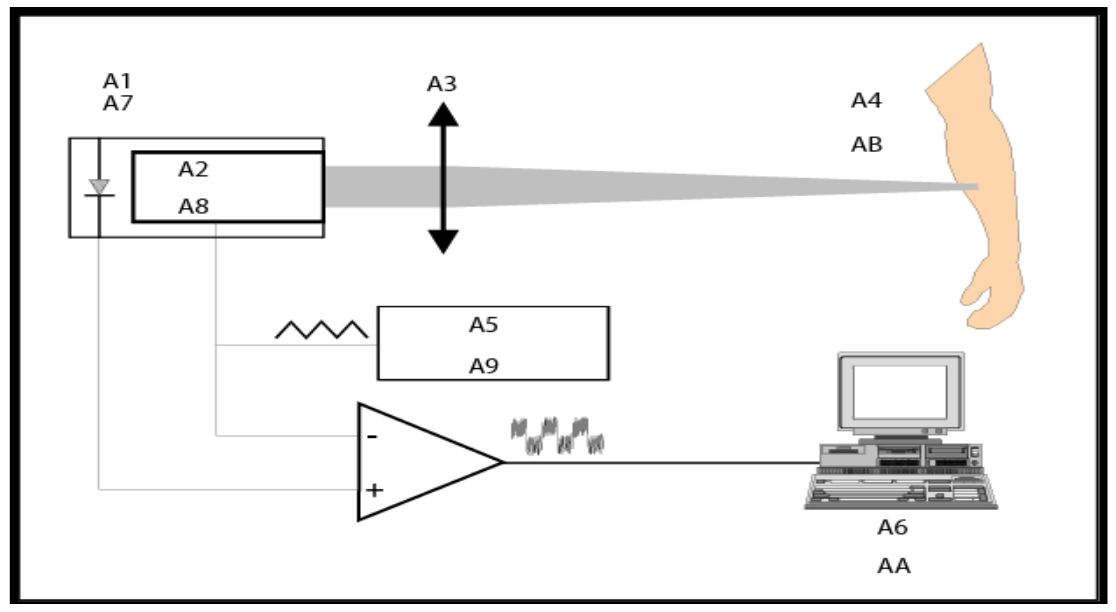

Figure 2: Schematic diagram of the experimental set-up.

modulating LF MMG signal as a composite signal. In a second step, the latter is filtered again using a severe $50 \mathrm{~Hz}$ Low pass filter which yields the desired low frequency Mechanomyogram signal. Then, this MMG signal is acquired and saved into the memory of a PC via cards interfaces with a sampling frequency of $1000 \mathrm{~Hz}$.

\section{Choi-Williams distribution}

Time and frequency covariance properties are considered as the most important desirable properties that an energy distribution should satisfy. This means that a time shift (or frequency) will induce the corresponding shift in the timefrequency plane (Spectrogram) [7]. The class of such distributions was defined and generalized by Cohen [8] and the general expression of each member of this class was given by:

$$
C_{x}(t, v, f)=\iiint_{-\infty}^{+\infty} e^{-j 2 \pi \xi(s-t)} f(\xi, \tau) x\left(s+\frac{\tau}{2}\right) x^{*}\left(s-\frac{\tau}{2}\right) e^{-j 2 \pi v \tau} d \xi d s d \tau
$$

where: $f(\xi, \tau)$ : is a two dimensional function called the parameterization function. This class is known as the Cohen's class. The advantages of such a unifying formulation are that it guarantees the possibility to obtain most of known energy distributions [7-9]. The parameterization function is also called the kernel function and referred as $\phi(\xi . \tau)$ which depends only on the product of the variables $\xi$ and $\tau$. This function is a decreasing one which considerably reduces the interferences terms which can blur the direct readability of the spectrogram. The Choi-Williams distribution is a member of the Cohen's class having a Gaussian kernel function expressed by: 


$$
f(\xi, \tau)=\phi(\xi, \tau)=e^{\left[-\frac{(\pi \xi \tau)^{2}}{2 \sigma^{2}}\right]}, \text { Choi-Williams kernel }[7,9] .
$$

Then the corresponding 2D_Choi-Williams distribution is given by [9]

$$
\mathrm{CW}_{\mathrm{x}}(\mathrm{t}, \mathrm{v})=\sqrt{\frac{2}{\pi}} \iint_{-\infty}^{+\infty} \frac{\sigma}{|\tau|} e^{-\frac{2 \sigma^{2}(s-t)^{2}}{\tau^{2}}} x\left(s+\frac{\tau}{2}\right) \cdot x^{*}\left(s-\frac{\tau}{2}\right) e^{-j 2 \pi v \tau} d s d \tau ;(4)
$$

where, $\sigma$ is a parameter that can be adjusted by proper software in order to reduce the cross terms in the $\mathrm{CW}$ spectrogram. These cross terms can be negative terms and blur the direct readability of most significant frequency components [7]. The value $\sigma$ can also be programmed to determine a good compromise between frequency and time resolutions. For more details, one should refer to references [7-9].

\section{Results and discussion}

Figures 3 and 4 represent the results obtained on two Normal subjects. They are organized so that for each MMG signal (a) acquired there exist two subwindows.

The first sub-window gives the display of the spectrogram of the corresponding Choi-Williams distribution (b). The second sub-window displays the Reassigned Morlet Wavelet Scalogram in order to compare and validate the results obtained with the Choi-Williams Spectrogram The protocol of MMG Signals measurement is that which had been used and detailed in references [2] and [3].

The subject is prepared in such a way that he can produce an isometric contraction necessary to generate the MMG signal. In the case of Normal 1 , it can be observed from Figure 3 that a good concentration of the most significant peaks of energy around and below $10 \mathrm{Hertz}$ in the $\mathrm{CW}$ spectrogram without being able to read precisely the most significant frequency.

Whereas, the Morlet Scalogram reveals that the frequency centroïd is clearly in $7 \mathrm{~Hz}$. For Normal 2, we can clearly notice the appearance of several most significant frequencies around $10 \mathrm{~Hz}, 12 \mathrm{~Hz}$, and $14 \mathrm{~Hz}$ from the $\mathrm{CW}$ spectrogram and this is clearly confirmed by the frequencies centroïds around the same frequencies in the Morlet Scalogram. It can be deduced from these second results that the isometric muscle contraction was more sustained by the second Normal who then excited more fast fibers and hence produced more high frequencies relatively to the MMG spectra bandwidth. This is probably due to a better adaptation to the required steps of the measurement protocol and the progressive grasping of the strain gauge.

The second set of results represented in Figure 5 and 6 concern the tests operated on the Patients under clinical environment with the consent of the hospital authorities. These patients were affected with certain different muscle disorders. The results are organized in the same manner as the Normal results. It 
(a)

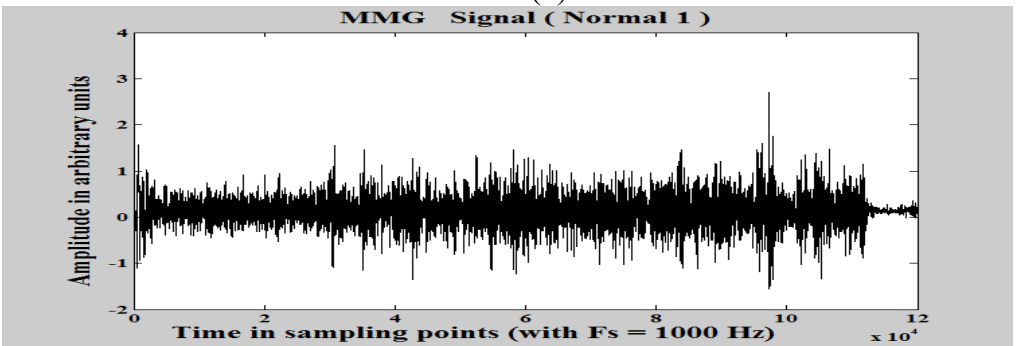

(b)

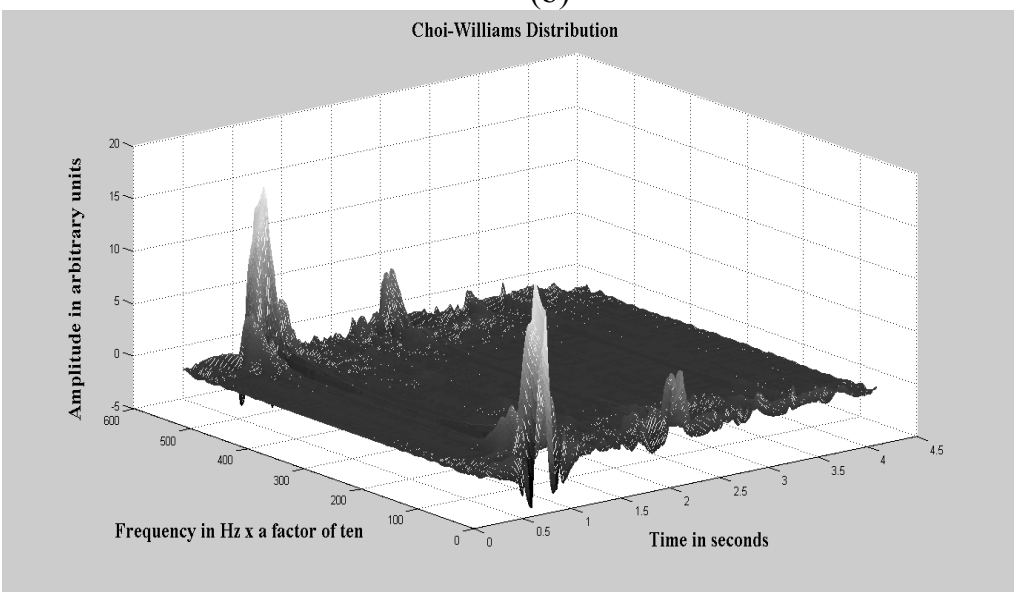

(c)

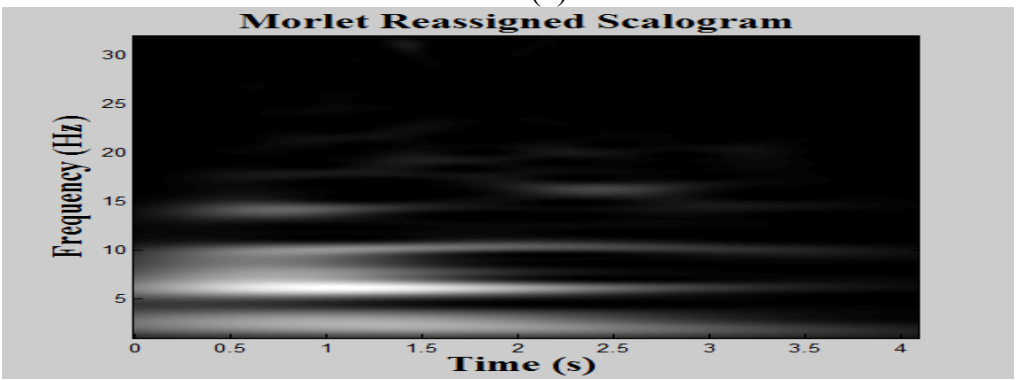

Figure 3: Results obtained on the first normal subject. (a) MMG signal, (b) Choi-Williams distribution, (c) Morlet Wavelet Scalogram. 
(a)

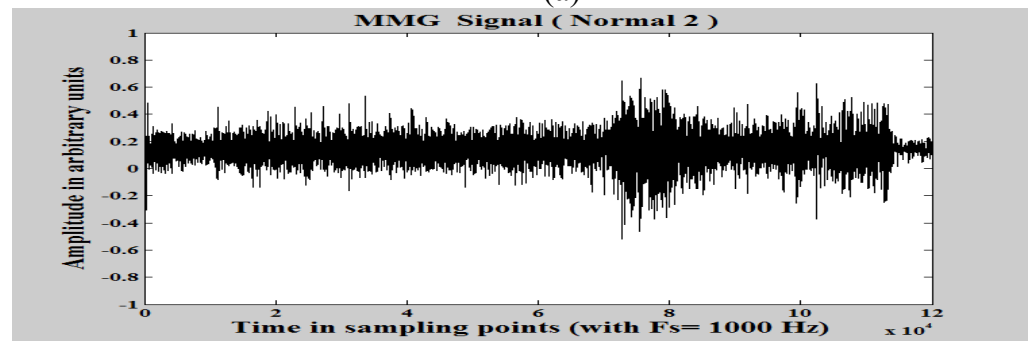

(b)

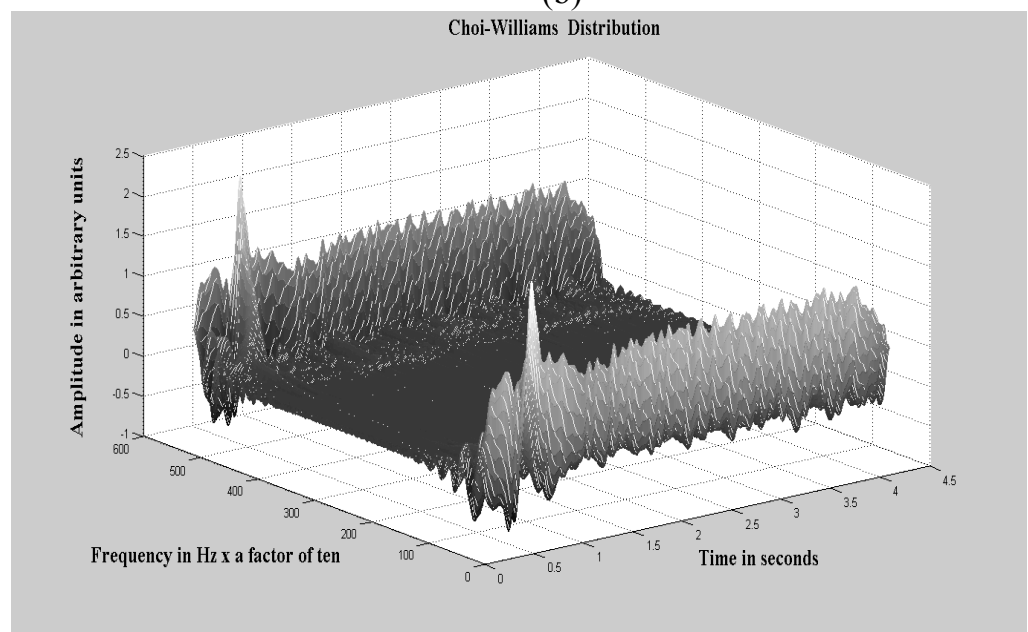

(c)

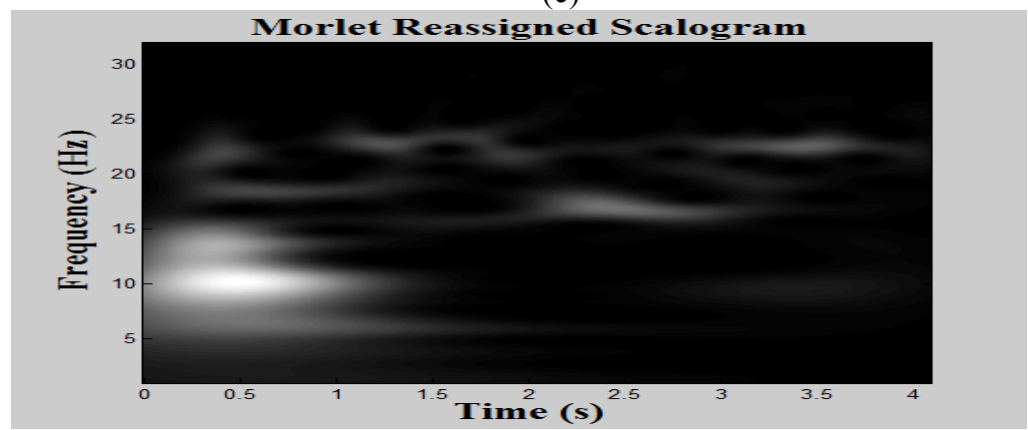

Figure 4: Results obtained on the second normal subject. (a) MMG signal, (b) Choi-Williams distribution, (c) Morlet Wavelet Scalogram. 
(a)

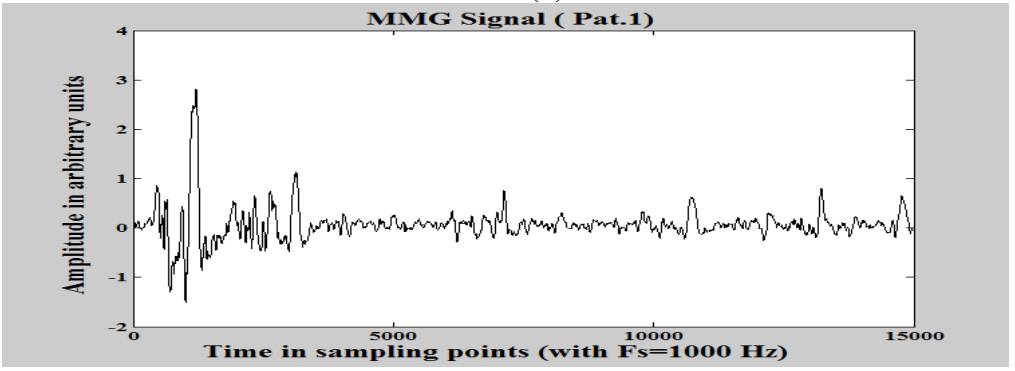

(b)

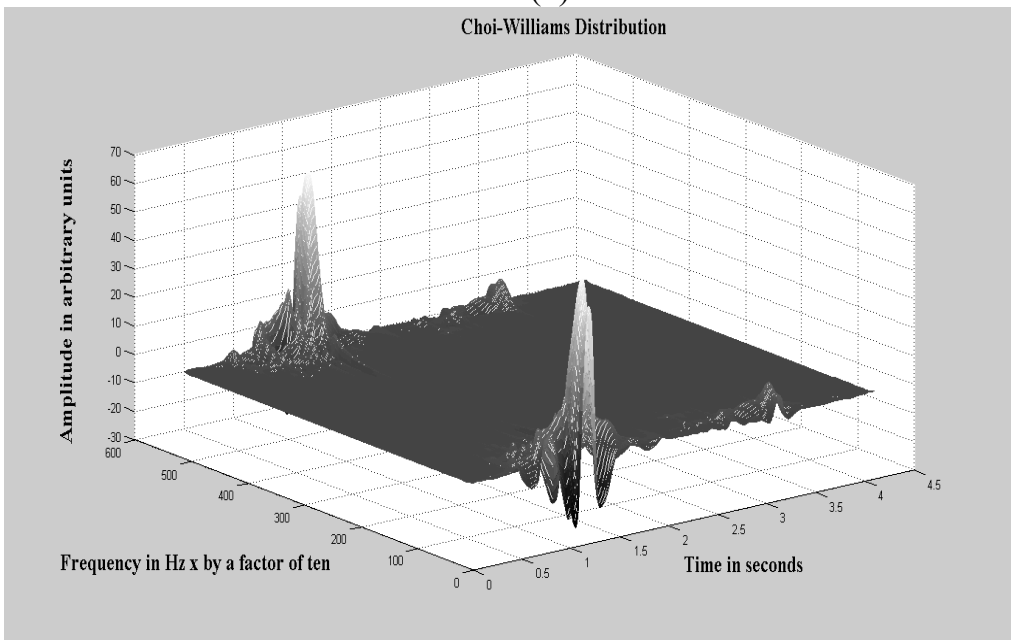

(c)

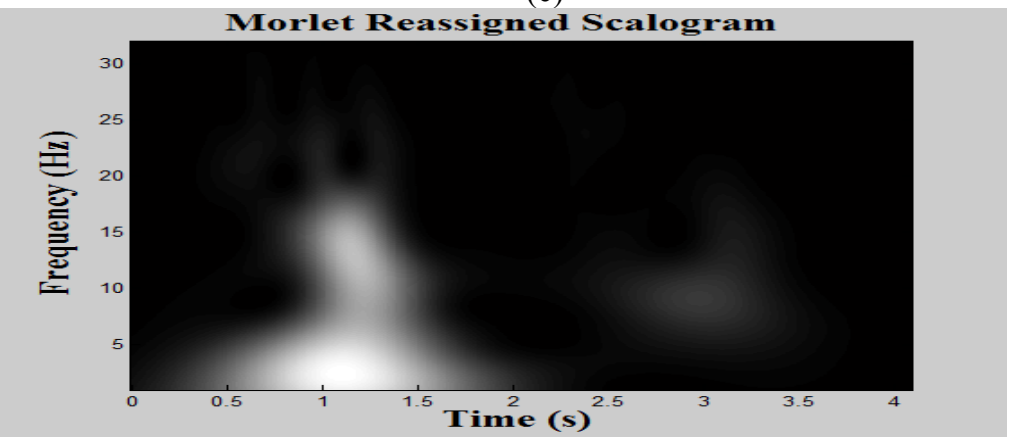

Figure 5: Results obtained on the first patient. (a) MMG signal, (b) ChoiWilliams distribution, (c) Morlet Wavelet Scalogram. 
(a)

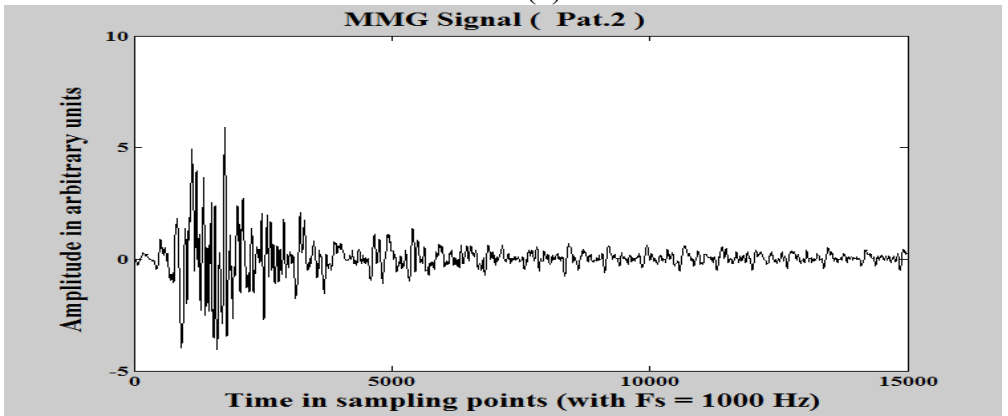

(b)

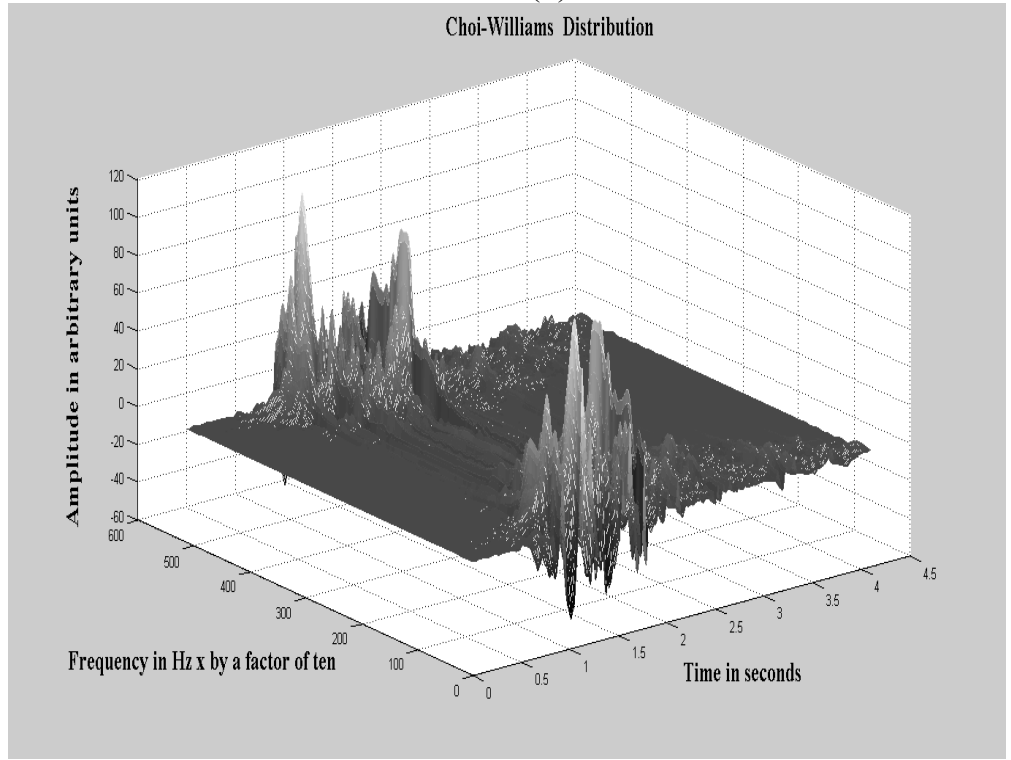

(c)

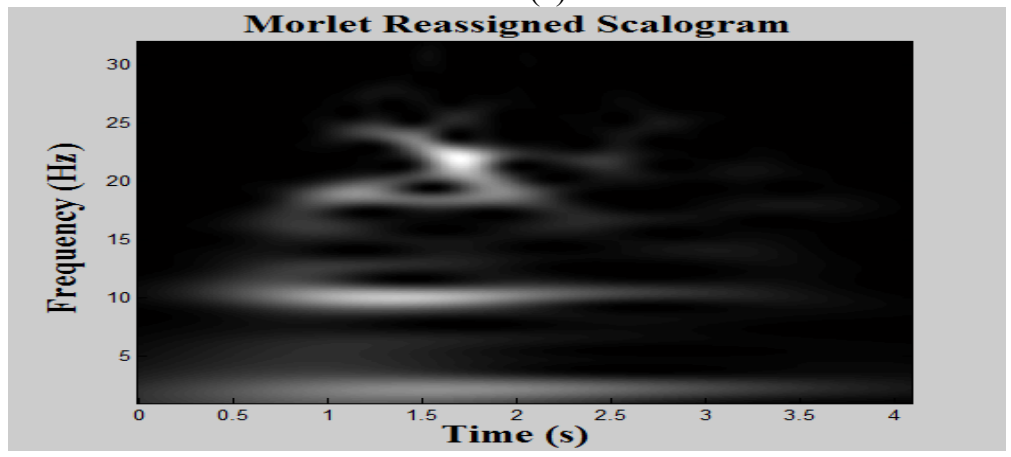

Figure 6: Results obtained on the second patient. (a) MMG signal, (b) ChoiWilliams distribution, (c) Morlet Wavelet Scalogram. 
can be seen from Figure 5 that Pat. 1 had developed a very feeble voluntary contraction in the first five (5) seconds of the MMG (a). This consequently implies last significant frequencies below $5 \mathrm{~Hz}$ in its $\mathrm{CW}$ corresponding representation and confusing frequencies around 12 and 14 Hertz. This fact is neatly confirmed by its Scalogram where the frequencies that appear neatly around $3 \mathrm{~Hz}$ and confusing frequencies around $12 \mathrm{~Hz}$. In this case, we can assert that this patient was more affected and the disease to be severe. It is obvious that this patient did his best to adjust and adapt his efforts to produce a voluntary isometric contraction but we got only tremors. Today, frequencies under $5 \mathrm{~Hz}$ are considered to be produced by tremors only.

In the case of Pat. 2 (Figure 6 hereafter) the second patient, it can be noticed that the amplitude of the MMG signal (Figure 6 (a)), is higher than in the case of Pat. 1 in the same first five (5) seconds period of the acquisition time. This denotes a better voluntary isometric contraction but not sustained after this period. Subsequently the Choi-Williams Spectrogram reveals few most significant frequencies around $20 \mathrm{~Hz}$ and many others confusing frequencies below that peak of energy which are hardly readable because of the presence of many blurring negative cross-terms.

It is obvious that these cross terms are smeared out in the Morlet Wavelet Scalogram (Figure 6 (c)) which is a strictly positive representation in the time frequency plane. Thus, its reassignment form is then considered as a smoothed version which smears out all the negative components. So the observations on the CW Spectrogram are clearly verified and observed with a good frequency resolution in the Scalogram which shows clear centroïds around $20 \mathrm{~Hz}$ and $10 \mathrm{~Hz}$ and few frequencies in the tremors range. It is obvious that this patient is affected by less severe disease. Also, as he showed a more improved adapted effort to the progressive constraints and requirements of the exercise sequence of the measurement protocol.

\section{Conclusion}

In this work, our main goal was to compare the efficiency of two Time Frequency Analysis tools namely the Choi-Williams distribution with the wellknown Reassigned Morlet Wavelet Scalogram. The CW is an energy distribution which belongs to the reputed Cohen's Class and hence satisfies many interesting and desired properties to analyze random and Non-stationary signals. It also offers the possibility to adjust and find a good compromise to perfectly localize the components of such a class of signal in time and frequency with appreciable good resolutions. The results obtained on the on Normal (Healthy) subjects were similar between both time frequency representations except that it remains some difficulties in reading correctly the frequencies in the $\mathrm{CW}$ spectrogram because of negative cross terms which blur the spectrogram and make hard this frequency readability. Whereas the Reassigned Morlet Scalogram reveals neatly these frequencies but it is very hard to localize these frequencies in time. This fact is also noticeable in the case of Patients where the $\mathrm{CW}$ was hardly readable to determine the most significant frequencies and also both representations have 
revealed the presence of tremors frequencies below $5 \mathrm{~Hz}$ and denoting a more or less severe muscle disorder.

So, it appears that the $\mathrm{CW}$ distribution spectrogram perfectly localizes the frequency components in time and hardly allows their determination in frequency because of remaining unavoidable negative cross terms which blur this readability. Whereas, in the case of the Reassigned Morlet Scalogram, Frequency components are precisely determined with a high frequency resolution but unfortunately poorly determined in time (bad time resolution).So, we can then conclude that both tools are powerful and complementary in Time Frequency Analysis of MMG signals but to be fully informative they should be used and implemented simultaneously to precisely determine the frequency components.

\section{References}

[1] A. Courteville, T. Gharbi, J.Y. Cornu, Non Contact Sensor based on the Optical Feedback in a Laser Diode, Journal of Biomedical Optics, Vol.3, N³, pp.281-285, 1998.

[2] S. Mekaoui, A. Houacine, T. Gharbi, Reassigned Spectrogram Representation for Mechanomyogram Signal Analysis, Journal of Sensor Letters, Vol.6, $\mathrm{N}^{\circ} 4$, pp.465-476, American Scientific Publishers, USA, 2008.

[3] S. Mekaoui, A. Houacine, T. Gharbi, Readability of Reassigned Scalograms and Extraction of Spectra Features for Signal Analysis, WIT Transactions on Biomedicine and Health, Vol.13, pp.287-300, WIT Press 2009, UK.

[4] T. Gharbi, D. Barchiesi, O Pergossi, H. Violand and C. Richard, Optical Near Field Data Analysis through Time-Frequency distributions. Application to the Characterization and Separation of the Image spectral content by the Reassignment, Journal of Optical Society of America, Vol. 27, $\mathrm{N}^{\circ} 12$, pp.2513-2519, USA, 1999.

[5] K. Peterman, Laser diode modulation and noise, editor: Kluwer Academic Publishers, Dordrecht, 1991.

[6] S. Shinohara, A. Mochizuki, H. Yoshida, M. Sumi, Laser Doppler Velocimeter using the Self Mixing Effect of a Semi Conductor Laser Diode, Journal of Applied Optics, Vol. 25, N 9, pp.1417-1419, 1996.

[7] H. I. Choi and W.J. Williams, Improved Time-frequency Representation of Multicomponent Signals using Exponential Kernels. IEEE Transactions on Acoustics and Signals Processing, Vol.37, N6, pp.862-871, June 1989.

[8] L. Cohen, Time-frequency distribution, A review, Invited paper in the proceedings of IEEE, Vol. 77, N7, pp.941-982, July 1989.

[9] F. Auger and P. Flandrin, Improving the Readability of the Time-Frequency and the Time-Scale Representations by the Reassignment Method. IEEE Transactions on Signal Processing, Vol.43, N5, pp.1068-1089, May 1995. 